\title{
Use of Implantable Pump Systems for Intraarterial, Intraventricular and Intratumoral Treatment of Malignant Brain Tumors
}

\author{
WILLIAM F. CHANDLER, ${ }^{a}$ HARRY S. GREENBERG, ${ }^{b}$ \\ WILLIAM D. ENSMINGER, ${ }^{c}$ RICHARD F. DIAZ, ${ }^{d}$ LARRY \\ R. JUNCK, ${ }^{b}$ TERRY W. HOOD, ${ }^{a}$ STEPHEN S. GEBARSKI, \\ AND MICHAELYN A. PAGE ${ }^{b}$ \\ ${ }^{a}$ Section of Neurosurgery \\ ${ }^{b}$ Department of Neurology \\ ${ }^{c}$ Department of Internal Medicine. \\ 'Department of Radiation Therapy \\ and \\ ${ }^{e}$ Department of Radiology \\ University of Michigan Medical Center \\ Ann Arbor, Michigan 48109
}

Significant advances in the development of implantable continuous infusion pumps have made available devices which are reliable and attractive for trials of regional chemotherapeutic treatment of various types of tumors involving the central nervous system. Since oftentimes tumors of the brain or CSF pathways respond in a dose-related fashion to either chemotherapy or radiation, it is reasonable to try to increase exposure on a regional basis, thus limiting systemic exposure and complications. It is this desire to increase regional exposure that has lead our group and others to utilize continuous infusion of various agents within the carotid arterial system, the ventricular system and even directly within the tumor itself. This paper will review our experience at the University of Michigan as well as the work of other investigators.

\section{INTRAARTERIAL PUMP INFUSIONS}

Continuous infusions of agents directly into the carotid artery for the regional treatment of brain tumors has certain inherent risks and to date has only been carried out on a very limited basis. Trials with this technique have either involved the infusion of cycle-specific chemotherapeutic drugs or the infusion of a radiosensitizer to enhance the effect of regional brain radiation. We have had experience in both of these areas and have used the same pump and technique for both.'

We have utilized the Infusaid (Infusaid Corp., Norwood, MA) implantable pump system in all cases. This pump weighs approximately 220 grams and has a side-port available for bolus drug infusions. The pump is driven by compressed freon gas within the charging chamber which is recharged with each routine filling of the drug chamber. The drug chamber holds $50 \mathrm{cc}$ of liquid and discharges the drug at a fixed predictable rate of 3 to $5 \mathrm{cc}$ per day at a pressure which is greater than arterial blood 
pressure. The pump is placed in a subcutaneous pocket just below the clavical and attached to the clavical with a heavy gauge suture. The outflow catheter is tunneled subcutaneously to the region of the carotid bifurcation and ultimately placed down the external carotid artery to the carotid bifurcation. The distal external carotid artery is then ligated, resulting in a strictly internal carotid artery infusion. With flow rates of only 3-5 cc per day, the high flow of the common carotid artery is not affected and there is no streaming effect within the carotid circulation. We have PET scan evidence that there is a uniform distribution of drug within the distribution of the internal carotid artery (unpublished data).

\section{Continuous Intraarterial Chemotherapy}

Prior to placement of the first human intracarotid pump, we tested the system in bilateral carotid arteries of two monkeys. ${ }^{\prime}$ The four pumps and catheters were left in place for 90 days and the carotid arteries were then studied with angiography and postmortem scanning electron microscopy. All vessels were patent and no significant endothelial abnormalities were noted. One monkey received unilateral $2^{\prime}$-deoxy5-fluorouridine (FUdR) at $0.1 \mathrm{mg} / \mathrm{kg} /$ day for 57 days and the other received dichloromethotrexate (DCMTX) at $0.2 \mathrm{mg} / \mathrm{kg} /$ days for 25 days. There were no apparent side effects related to the chemotherapy.

Following the animal experiment, intracarotid pumps were placed in six patients with malignant gliomas in the distribution of a single carotid artery. The plan with these patients was to use the side-port of the pump for bolus intraarterial injections of cycle nonspecific BCNU every six weeks and in selected patients to use the pump for continuous infusions of cell cycle specific agents. The logic being that for cycle specific agents to be effective they must be present continuously throughout the cell cycle. The agents tested were FUdR and DCMTX. One patient received $6.5 \mathrm{mg} /$ day of FUdR continuously for 70 days as well as DCMTX, $3 \mathrm{mg} /$ day for 25 days, with no apparent adverse effects. A second patient received FUdR, $4.8 \mathrm{mg} /$ day, for 14 days and developed a mild dermatitis on the forehead. The drug was stopped and the dermatitis cleared. All six patients received from one to four courses of intraarterial BCNU, 200 $\mathrm{mg} / \mathrm{m}^{2}$, via the side-port. There were no new neurologic deficits attributable to the implanted catheter or the pump system and there were no infections related to the pump. The pumps remained in these patients until death and were functional for up to 3 years. Carotid angiograms were performed on several patients with the catheters in place and no intraluminal defects were noted. Postmortem examination of several carotids did not reveal any adverse endothelial changes. It is not possible to make meaningful comments regarding survival, since only two of the patients received continuous infusions of chemotherapeutic agents, but we can say that the intracarotid pump system functioned effectively and safely for extended periods of time.

Kindt, who was originally part of our group, carried out similar experimental work with colleagues at the University of Colorado. ${ }^{2}$ They used the same Infusaid pumps and the same technique for catheter placement. They placed the pumps in six patients and infused FUdR at doses ranging from 0.5 to $2.5 \mathrm{mg}$ /day for a mean of 31 days. They also infused BCNU via the side-port in all patients in doses of $\mathbf{4 0 0} \mathrm{mg}$ every six weeks. They noted ipsilateral mucositis and conjunctivitis in the distribution of the ophthalmic artery. They believed this was the dose limiting factor of FUdR and found that any dose beyond $1 \mathrm{mg} /$ day resulted in these complications. None of the six patients responded and all died of their malignant gliomas within 30 weeks of the study. They concluded that the infusion system worked well and that the maximum tolerable dose of FUdR was $1.0 \mathrm{mg} / \mathrm{day}$. 
Overall, the experience at Michigan and Colorado would suggest that the Infusaid implantable continuous pump system can be used safely and effectively to deliver chemotherapeutic agents directly into the carotid circulation. Unfortunately, the ideal agent for this type of infusion is not currently available.

\section{Continuous Intraarterial Radiosensitization}

It is well known that malignant gliomas respond to external beam radiation therapy. Since it is known that the response is dose related, it would be desirable either to increase the actual dose to the tumor or in some way to make the given dose more effective. Various techniques including radioactive implants are being used to safely increase the dose to the tumor and radiosensitizers have become available to increase effectiveness. BUdR is such a radiosensitizer and increases the radiation effect by a factor of about two. Since BUdR is rapidly cleared by the body, it has been demonstrated that the regional intraarterial route of administration provides a higher exposure to the tumor. BUdR is a thymidine analog and thus will be taken up in a competitive manner with thymidine making cells more vulnerable to radiation exposure. For this reason it is given by continuous infusion beginning two weeks prior to radiation therapy and concommitantly with the entire course of external beam radiation therapy. Efforts by $\mathrm{Hoshino}^{3}$ using BUdR via an external intracarotid pumping system suggested prolonged survival, but there were complications related to the bedside pumping system. With our above experience demonstrating a safe implantable continuous intracarotid pumping system, we developed a protocol to treat a series of patients with malignant gliomas with BUdR as a radiosensitizer for external beam radiation therapy.

Prior to initiating clinical trials of intracarotid BUdR we carried out a toxicity study using monkeys with unilaterally implanted intracarotid pump systems. Six monkeys were used, with three receiving $B U d R, 10 \mathrm{mg} / \mathrm{kg} / \mathrm{day}$, and three receiving only heparinized saline. They all received concommitant whole brain radiation with $6000 \mathrm{cGy}$ of Cobalt 60 external beam radiation. The animals tolerated the treatment well and there were no differences pathologically between the BUdR and saline infused hemispheres. There were small areas of focal necrosis in both hemispheres.

To date we have treated 18 patients with intraarterial BUdR, all using the identical intracarotid infusion system described above. The average age of the patients was 47.6 years (range 20-71 years) and the current follow-up time averages 11 months with a maximum of 23 months. Three of the patients had grade III astrocytomas and fifteen patients had grade IV. All patients received BUdR on a continuous basis for 2 weeks prior to radiation and all during the radiation therapy. Patients received 5,640 cGy over 6 weeks. The dose of BUdR ranged from 400 to $600 \mathrm{mg} / \mathrm{m}^{2} /$ day, with 5 receiving $400 \mathrm{mg} / \mathrm{m}^{2} /$ day, 1 receiving $450 \mathrm{mg} / \mathrm{m}^{2} /$ day, 7 receiving $500 \mathrm{mg} / \mathrm{m}^{2} /$ day, and 5 receiving $600 \mathrm{mg} / \mathrm{m}^{2} /$ day. The side effects and toxicities are listed in TABLE 1 and became more frequent and more severe with higher doses of BUdR. The most severe side effects involved the ipsilateral orbit and forehead, including blepharitis, iritis, conjunctivitis and dry and moist desquamation of the skin. One patient required enucleation of the ipsilateral eye following radiation therapy. One patient developed a Stevens-Johnson syndrome requiring cessation of BUdR and radiotherapy treatment. One patient required transfusion of packed rbc's and platelets because of myelosuppression. Most of the side effects listed in TABLE 1 began to subside within one week of completing radiation therapy and usually returned to normal within 6 to 12 weeks posttreatment. 
Although it is too early in the follow-up of these 18 patients to derive meaningful survival data, some preliminary observations can be made. Of the 8 patients that have been followed 12 months or longer 2 have died, 3 have progressed, 1 is stable and 2 appear disease free at 21 and 22 months posttreatment. In the overall group of 18 , tumor progression has been verified in 11 and 6 have died. Intraarterial chemotherapy with BCNU by bolus infusion through the pump side-port has been added to the treatment of 5 of those patients with disease progression. Partial responses were seen in 3 of these and no response was evident in 2.

\section{INTRAVENTRICULAR PUMP INFUSIONS}

Regional treatment of certain neoplasms of the central nervous system via the CSF pathways is attractive for a number of reasons. Since the subarachnoid space is a sanctuary from the systemic circulation because of the blood-brain barrier, metastatic tumors in the leptomeningeal spaces may grow without adequate exposure to systemic chemotherapeutic treatments. On the other hand, agents administered intrathecally may achieve very high exposures with little or no systemic toxicity. Partly because of

TABLE 1. Side Effects and Toxicities of BUdR and External Beam Radiation Therapy, 18 Patients

\begin{tabular}{lccc}
\hline & Mild & Moderate & Severe \\
\hline Anorexia/Altered taste & 4 & 9 & 5 \\
Weight Loss & 5 & 9 & 1 \\
Blepharitis/Iritis & 6 & 8 & 4 \\
Forehead Dermatitis & 3 & 8 & 7 \\
Systemic Dermatitis & 1 & 0 & 1 \\
Fatigue & 1 & 10 & 7 \\
Myelosuppression & 3 & 1 & 0 \\
Nail Changes (onychomadeis) & 18 & 0 & 0 \\
\hline
\end{tabular}

the longer survivals with the current systemic treatment of many neoplasms, we are seeing an increase in leptomeningeal metastases. Patients may present with cortical, cranial nerve or spinal symptoms, with $15 \%$ having seizures and $20 \%$ having hydrocephalus. ${ }^{4}$ Breast, lung and melanoma remain the most common primaries, but with a long list of other cell types. Untreated, the survival is only 6 weeks. The current standard treatment regimen is intermittent doses of intrathecal chemotherapy plus radiation therapy. The drugs commonly utilized for this are cycle specific antimetabolites methotrexate (MTX) and cytosine arabinoside (Ara-C) or cycle nonspecific Thio-TEPA. Since it has been demonstrated that intraventricular injections provide a more even CSF distribution than lumbar injections, most treating physicians utilize a ventricular catheter and reservoir for intrathecal treatment.

In spite of the improved survival of 4 to 6 months with $12 \%$ living longer than one year with intermittent intrathecal chemotherapy plus radiation, problems remain with this approach. With intermittent bolus doses there are peaks and troughs which both lessen the effectiveness of treatment and increase the risk of toxicity. Bleyer ${ }^{5}$ has shown that the leukoencephalopathy seen with intrathecal MTX treatment is related to the high drug concentration within the CSF. Bleyer" suggested in 1978 that a "concentra- 
tion times time" (CXT) method be used in which lower peak concentrations would be achieved, but more consistently and for a longer time. If cycle specific drugs are used then the exposure time must be prolonged. In 1980 we decided to utilize the Infusaid continuous infusion pump to achieve this goal of a steady state CSF concentration over a prolonged period of time.

As in the arterial studies described above, we first developed a monkey model to test toxicity and to carry out pharmacokinetic studies to determine steady state CSF drug levels. With this information, we then placed the Infusaid pumps in 13 patients with the infusion catheters connected to a side connector of a ventricular reservoir. ${ }^{6}$ In each case the ventricular catheter and reservoir had been previously implanted under local anesthetic and a radionuclide cisternogram carried out to assure normal bulk flow of CSF. Once the pump was added to the system the reservoir provided access to the CSF for measuring drug concentrations. Either MTX or FUdR were administered continously via the pump system. When MTX was given at $0.5 \mathrm{mg} /$ day CSF levels ranged between 1.8 and $2.5 \mu \mathrm{M}$ and when MTX was given at $2 \mathrm{mg} /$ day CSF levels were $12( \pm 2) \mu \mathrm{M}$. The cytotoxic level for leukemia cells is $0.5 \mu \mathrm{M}$. Relatively constant MTX levels were maintained throughout a given 7-14 day infusion cycle. Serum MTX levels were in every case less than $0.01 \mu \mathrm{M}$, the lower limit of the sensitivity of the assay. Thus the sustained regional exposure in the CSF was 200 to 3,000 times the systemic exposure. It should be mentioned that concentration is related to the bulk flow of the CSF and that it may be altered with changes in intracranial pressure. Also the blood-brain barrier may be "opened" in regions by the tumor and thus more drug may be lost from the CSF, affecting concentration.

A total of 13 patients underwent placement of the ventricular infusion system. Five of the early patients had solid intracerebral malignant gliomas and were given MTX. None of these patients lived more than 6 months and only 3 had minimal evidence of response. It was concluded that this is not an effective treatment for intracerebral tumors. The other 8 patients had leptomeningeal carcinomatosis related to a primary neoplasm elsewhere ( 5 breast, 1 lymphoma, 1 leukemia, and 1 lung). Three were treated with MTX and all responded for from 2 to 12 months before dying of their systemic disease. Five were treated with FUdR and 2 responded for from 1 to 15 months before death from systemic progression. Both of these responders had previously failed to respond to intermittent doses of MTX. There were no pumprelated infections and no pump failures. One early patient developed transverse myelitis presumably related to the preservative in the MTX. Since then only preservative free MTX was used. Several patients developed mild meningismus and fever within 24 hours of initiation of infusion, but this was easily suppressed with steroids. Three patients developed subclinical leukoencephalopathy as seen on CT scanning. This has been described in patients with intrathecal chemotherapy, often in association with radiotherapy.

Beck and Hochberg ${ }^{4}$ in 1985 reported using the same Infusaid intraventricular infusion system in 4 patients with leptomeningeal tumors. They used preservative free MTX, 20-25 mg/week, to achieve CSF levels of 1-20 $\mu \mathrm{M}$. Two patients had breast cancer, one had multiple myeloma and one had an esthesioneuroblastoma. One died at 6 months with leukoencephalopathy and residual tumor, and one recurred at 3 months. One died of systemic disease at 3 months, and in the fourth patient the pump became blocked after just a few weeks and was never replaced because of the poor condition of the patient.

Several other potential indications for continuous intraventricular infusion have been explored in recent years. Harbaugh et $a l^{7}$ have used intraventricular infusions of the acetylcholine agonist bethanechol in an attempt to treat Alzheimer's disease, since there is an apparent deficiency of acetylcholine in this disease. Several investigators 
are pursuing the possibility of using intraventricular L-dopa in the treatment of Parkinson's disease. This would avoid the peripheral effects of L-dopa and provide a predictable level available to the brain. Thyrotropin-releasing hormone, which does not cross the blood-brain barrier, is currently being tested intraventricularly as a possible agent to decrease the progressive loss of muscle function in amyotrophic lateral sclerosis.

\section{INTRATUMORAL PUMP INFUSIONS}

Since we have not had personal experience with intratumoral infusion techniques and this subject is covered by Bouvier $e t$ al. in the following paper, this technique will only be briefly mentioned. In an attempt to circumvent the natural obstruction of the blood-brain barrier to hydrophilic drugs researchers have infused chemotherapeutic agents directly into malignant brain tumors. To study the ability of drugs to diffuse through the normal brain, Kroin and Penn ${ }^{8}$ used an osmotic minipump cisplatin into the cerebellum and frontal cortex of rats. They found that therapeutic concentrations of cisplatin could be achieved in a volume of $1 \mathrm{~cm}^{3}$ using this type of pump. Penn and co-workers ${ }^{9}$ then developed a cerebellar tumor model in rats in which they were able to use the osmotic minipump to infuse either cisplatin $(0.5 \mathrm{mg} / \mathrm{ml})$ or fluorouracil $(25$ $\mathrm{mg} / \mathrm{ml}$ ) at $0.9 \mu \mathrm{l} /$ hour for 7 days within the tumor. They showed an increase in survival time for each drug as compared to control saline infusions. Bouvier et al ${ }^{10}$ then used the same osmostic minipump system to infuse cisplatin $(1 \mathrm{mg} / \mathrm{ml})$ into the malignant glioma of a patient via multiple stereotaxically placed intratumoral catheters. Each catheter bundle contained 4 smaller catheters. Thus by placing 17 catheter bundles in the tumor, a total of 68 small infusion catheters were active. The catheters were left in place for a total of 10 days, infusing a total of $0.82 \mathrm{mg} /$ day of cisplatin. Bouvier stated that a comparable systemic dose would be $36 \mathrm{mg} / \mathrm{day}$; thus there was a marked reduction in systemic exposure to a potentially toxic agent. The patient died within 6 months of tumor progression, but tolerated the infusion well. This report demonstrates that continuous intratumoral infusion of a sizable tumor is now feasible.

\section{REFERENCES}

1. Phillips, T. W., W. F. Chandler, G. W. Kindt, W. D. Ensminger, H. S. Greengerg, J. F. SEEGER, K. M. DOAN \& J. W. Gyves. 1981. New implantable continuous administration and bolus dose intracarotid drug delivery system for the treatment of malignant gliomas. Neurosurgery 11: 213-218.

2. Mughal, T. I., L. M. Glode, T. J. Braun, W. Klingensmith, J. M. Geier \& G. W. KINDT. 1986. Phase I clinical trial of intracarotid bis-chloroethylnitrosourea (BCNU) and 2'-deoxy-5-fluorouridine (FUDR) in malignant astrocytomas. J. Neuro-Oncol. 3: 291-296.

3. Hosino, T. 1974. Radiosensitization of brain tumors. In Modern Radiotherapy and Oncology: CNS Tumors. T. J. Deeley, Ed. 170-184. Butterworth. London.

4. BECK, D. O. \& F. H. HOCHBERG. 1986. Intraventricular chemotherapy for leptomeningeal carcinoma. In Cancer Chemotherapy by Infusion. J. J. Lokich, Ed. Precept Press. Chicago, IL.

5. Bleyer, W. A., G. D. Poplak, A. R. Simon, et al. 1978. "Concentration x time" methotrexate via subcutaneous reservoir: a less toxic regimen for intraventricular chemotherapy of central nervous system neoplasms. Blood 51: 835-842.

6. Dakil, S., W. D. Ensminger, G. W. Kindt, J. Niederhuber, W. F. Chandler, H. S. 
GREeNBERG \& R. WheELER. 1981. Implanted system for intraventricular drug infusion in central nervous system tumors. Ca. Treat. Reports 65: 401-411.

7. Harbaugh, R. E., D. W. Roberts, D. W. Combs, et al 1984. Preliminary report: intracranial cholinergic drug infusion in patients with Alzheimer's disease. Neurosurgery 15: $514-518$.

8. KROIN, J. S. \& R. D. PENN. 1982. Intracerebral chemotherapy: chronic microinfusion of cisplatin. Neurosurgery 10: 349-354.

9. Penn, R. D., J. S. Kroin, J. E. Harris, K. M. Chiu \& D. P. Braun. 1983. Chronic intratumoral chemotherapy of a rat tumor with cisplatin and fluorouracil. Appl. Neu rophysiol. 46: 240-244.

10. Bouvier, G., R. D. PenN, J. S. Kroin, R. Belque \& M. J. Guerard. 1987. Direct delivery of medication into a brain tumor through multiple chronically implanted catheters. Neurosurgery 20: 286-291. 